\title{
Vibronic Coherence in the Charge Separation Process of the Rhodobacter sphaeroides Reaction Center
}

\author{
Fei Ma, ${ }^{*}{ }^{\dagger}$ Elisabet Romero, ${ }^{\dagger}$ Michael R. Jones, ${ }^{\ddagger}$ Vladimir I. Novoderezhkin, ${ }^{\S}$ and Rienk van Grondelle ${ }^{\dagger}$
}

${ }^{\dagger}$ Department of Physics and Astronomy, Faculty of Sciences, VU University Amsterdam, De Boelelaan 1081, 1081 HV Amsterdam, The Netherlands

${ }^{\ddagger}$ School of Biochemistry, University of Bristol, Biomedical Sciences Building, University Walk, Bristol BS8 1TD, United Kingdom

${ }^{\S}$ A. N. Belozersky Institute of Physico-Chemical Biology, Moscow State University, Leninskie Gory, 119992 Moscow, Russia

Supporting Information

ABSTRACT: Two-dimensional electronic spectroscopy was applied to a variant of the reaction center (RC) of purple bacterium Rhodobacter sphaeroides lacking the primary acceptor ubiquinone in order to understand the ultrafast separation and transfer of charge between the bacteriochlorin cofactors. For the first time, characteristic $2 \mathrm{D}$ spectra were obtained for the participating excited and charge-transfer states, and the electron-transfer cascade (including two different channels, the $\mathrm{P}^{*}$ and $\mathrm{B}^{*}$ channels) was fully mapped. By analyzing quantum beats using $2 \mathrm{D}$ frequency maps, excited-state vibrational modes at 153 and $33 \mathrm{~cm}^{-1}$ were identified. We speculate that these modes couple to the charge separation (CS) process and collectively optimize the CS and are responsible for the superhigh efficiency.

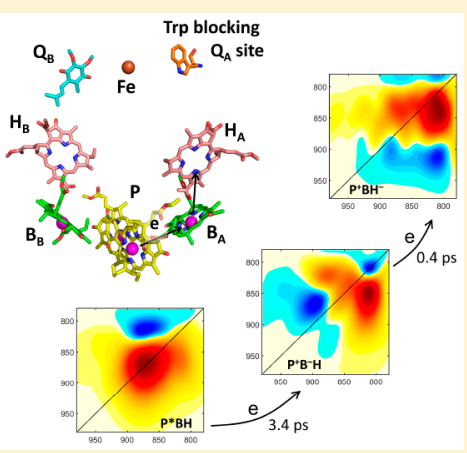

$\mathrm{R}$ eaction center (RC) pigment-proteins facilitate the key reaction of photosynthesis in which harvested solar energy is used to power separation of electrical charge across a lipid bilayer membrane. ${ }^{1,2}$ Many of the fundamental aspects of ultrafast light-powered charge separation (CS) have been unraveled in the relatively simple RC from the purple bacterium Rhodobacter (Rba.) sphaeroides. The bacteriochlorin cofactors of this RC are a pair of bacteriochlorophyll (BChl) $a$ (P), two monomeric BChl $a$ (B), and two bacteriopheophytin $a$ $(\mathrm{H})$ molecules arranged in two branches around an axis of quasi-two-fold symmetry (Figure 1$){ }^{3} \mathrm{CS}$ is initiated from the first singlet excited state of $\mathrm{P}\left(\mathrm{P}^{*}\right)$, an electron being transferred to a ubiquinone $\left(Q_{A}\right)$ via the intervening $B_{A}$ and $\mathrm{H}_{\mathrm{A}}$ that make up the "active branch" or "A-branch" of cofactors. ${ }^{4}$ At room temperature, the $\mathrm{P}^{*} \rightarrow \mathrm{P}^{+} \mathrm{B}_{\mathrm{A}}^{-}, \mathrm{P}^{+} \mathrm{B}_{\mathrm{A}}^{-} \rightarrow$ $\mathrm{P}^{+} \mathrm{H}_{\mathrm{A}}^{-}$, and $\mathrm{P}^{+} \mathrm{H}_{\mathrm{A}}^{-} \rightarrow \mathrm{P}^{+} \mathrm{Q}_{\mathrm{A}}^{-}$electron-transfer (ET) steps occur with time constants of 3-5, 0.5-1, and 200 ps, respectively. ${ }^{1,5}$ CS can also occur via another ET channel, $\mathrm{B}^{*} \rightarrow \mathrm{P}^{+} \mathrm{B}_{\mathrm{A}}^{-}$, with time constants of $0.2-0.5$ ps. ${ }^{6,7}$ The CS process proceeds with a near-unity quantum efficiency.

Coherent two-dimensional electronic spectroscopy (2DES) has developed into a powerful tool for the study of excitation energy transfer (EET) and ET dynamics in photosynthetic systems. $^{8-20}$ The related Photosystem II RC from oxygenic phototrophs has been studied by $2 \mathrm{DES},{ }^{14,15}$ but the ET cascades were difficult to resolve because the absorption bands of the chlorin cofactors strongly overlap, producing high signal congestion. In contrast the Rba. sphaeroides RC exhibits neardiscrete absorption bands with maxima at 865,805 , and $760 \mathrm{~nm}$ attributable to the $\mathrm{P}$ pair, $\mathrm{B}_{\mathrm{A}} / \mathrm{B}_{\mathrm{B}}$, and $\mathrm{H}_{\mathrm{A}} / \mathrm{H}_{\mathrm{B}}$, respectively. This

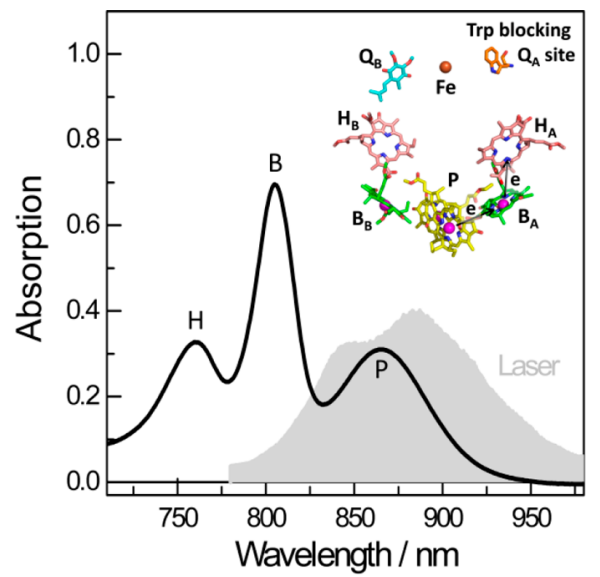

Figure 1. Room-temperature linear absorption spectrum of the AM260W RC (black) overlaid with the laser spectrum (gray). The inset shows the arrangement of cofactors and the ET pathway in the AM260W RC, which lacks a $Q_{A}$ acceptor due to an Ala to $\operatorname{Trp}$ replacement.

raises the prospect that $2 \mathrm{DES}$ can resolve details of the ET kinetics in bacterial RCs, a possibility supported by modeling. ${ }^{21}$

To date, 2DES studies performed on Rba. sphaeroides RCs have exclusively probed EET processes in complexes where $\mathrm{P}$ was oxidized to $\mathrm{P}^{+11,22,23}$ or kept reduced, ${ }^{24}$ and direct

Received: January 11, 2018

Accepted: March 27, 2018

Published: March 27, 2018 


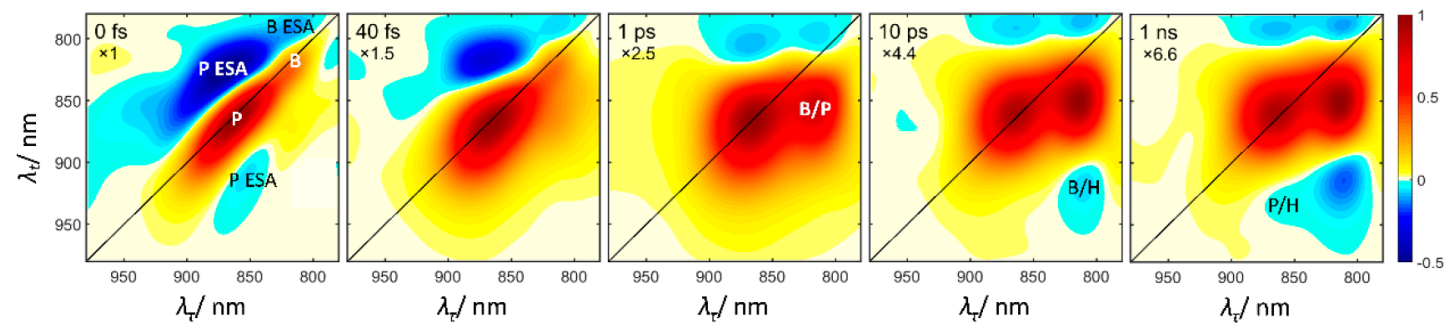

Figure 2. Absorptive total 2D spectra of the AM260W RC at the indicated population time T. Symbols $\lambda_{\tau}$ and $\lambda_{t}$ denote the excitation and detection wavelengths, respectively. The spectra are normalized to the maximum of the diagonal $\mathrm{P}$ signal; the relative amplitude multiplier is shown below the $T$. The identities of particular signals are indicated in each of the spectra: diagonal peaks are labeled with a single symbol designating the corresponding state; cross peaks are labeled using two symbols, with the first representing the locally excited state and the second representing the acceptor state.

observation of the ET cascade has not been carried out. A complication in applying $2 \mathrm{DES}$ to native $\mathrm{Rba}$. sphaeroides RCs is that the final states $\mathrm{P}^{+} \mathrm{Q}_{\mathrm{A}}^{-}$and $\mathrm{P}^{+} \mathrm{Q}_{\mathrm{B}}^{-}$have recombination lifetimes on the order of millseconds to seconds, such that $\mathrm{P}$ spends significant periods in the photo-oxidized state and is unable to be excited and to perform $\mathrm{CS}^{25}$ In this work, we overcame this problem by using an engineered $\mathrm{RC}$ in which an alanine at residue 260 of the M-polypeptide is replaced by tryptophan (AM260W). This mutation causes the RC to assemble without a $Q_{A}$ ubiquinone ${ }^{26,27}$ such that the forward $\mathrm{P}^{+} \mathrm{H}_{\mathrm{A}}^{-} \rightarrow \mathrm{P}^{+} \mathrm{Q}_{\mathrm{A}}^{-}$ET step is blocked and the majority of $\mathrm{P}^{+} \mathrm{H}_{\mathrm{A}}^{-}$ decays to the ground state with a lifetime of $\sim 17 \mathrm{~ns}^{28}$ As a result, no inactive $\mathrm{RCs}$ with an oxidized $\mathrm{P}$ persist beyond the repetition time between excitations ( $1 \mathrm{~ms}$ in this work), but the $\mathrm{P}^{*} \rightarrow \mathrm{P}^{+} \mathrm{B}_{\mathrm{A}}^{-} \rightarrow \mathrm{P}^{+} \mathrm{H}_{\mathrm{A}}^{-}$ET rates are essentially identical to those in the wild-type $\mathrm{RC}^{29}$

Potentially important aspects of the mechanism of CS in the $R b a$. sphaeroides $\mathrm{RC}$ are quantum effects, i.e., quantum beating arising from coupling between electronic, vibrational, or mixed (vibronic) states. Previous pump-probe experiments have found a rich structure of oscillations, ${ }^{30-33}$ which were thought to play a role in efficient ET. ${ }^{34}$ In this work, we used $2 \mathrm{DES}$ to distinguish each CT intermediate along the two ET pathways of the neutral AM260W RC and tried to understand the role of the long-lived quantum beats in CS.

The excitation pulse predominantly excited $\mathrm{P}$ with some minor excitation of $\mathrm{B}$ (Figure 1). The absorptive 2D spectra at five population times $(T)$ are shown in Figure 2. Signals associated with the $\mathrm{P} \rightarrow \mathrm{P}^{*}$ transition, the positive ground-state bleaching (GSB) and stimulated emission (SE) on the diagonal, and the negative excited-state absorption (ESA) off the diagonal, appeared simultaneously following excitation. At the same time, small GSB and ESA signals attributable to $\mathrm{B}^{*}$ also appeared, and a small cross peak centered at $(815,870) \mathrm{nm}$ ((excitation wavelength, detection wavelength)) was visible. This cross peak may reflect dynamic coherence between $\mathrm{P}^{*}$ and $\mathrm{B}^{*}$ at $\mathrm{T}=0 \mathrm{fs}$, and later, its growth may reflect two processes: EET or/and ET from $\mathrm{B}^{*}$ to $\mathrm{P}$. Both processes were fast; the EET was complete in around $200 \mathrm{fs},{ }^{1,25}$ and the ET had a time constant of $0.2-0.5$ ps. $^{6,7}$ The $2 \mathrm{D}$ spectrum at $T=0$ fs allowed an upper estimate of the homogeneous line width of the exciton between the ground and excited states, which was $340 \mathrm{~cm}^{-1}$ $(2 \gamma)$ determined by the full width at half-maximum of the antidiagonal broadening of the P GSB band. $\gamma$ is the dephasing rate and is inversely proportional to the coherence lifetime; ${ }^{19}$ thus, the electronic coherence lifetime was estimated to be 54 fs. The P GSB decreased significantly during the first $50 \mathrm{fs}$, and its shape became more rounded. This spectral diffusion reflected redistribution of the excitation energy among the exciton state manifold coupled to the protein bath.

Three different cross peaks appeared on a picosecond time scale. The positive one centered at $(815,855) \mathrm{nm}$ arose with a time constant of $430 \mathrm{fs}$. It is slower than the $\mathrm{B}^{*} \rightarrow$ P EET $(200$ fs); therefore, most probably it corresponded to $\mathrm{B}^{*} \rightarrow \mathrm{P}^{+} \mathrm{B}_{\mathrm{A}}^{-} \mathrm{ET}$ (labeled " $\mathrm{B} / \mathrm{P}$ ") and thus was a feature of the $\mathrm{P}^{+} \mathrm{B}_{\mathrm{A}}^{-} \mathrm{CT}$ state. The remaining two cross peaks were negative and centered at $(810,920)$ and $(865,927) \mathrm{nm}$. They formed nearly synchronously on a time scale of several picoseconds, although the weak $(865,927) \mathrm{nm}$ cross peak was partially obscured by the $\mathrm{P}$ GSB signal and therefore seemed to appear later. According to previous pump-probe experiments, the positive signal at 920-930 $\mathrm{nm}$ can be attributed to the absorption of $\mathrm{H}^{-}$, with the absorption of $\mathrm{B}^{-}$centered at $1025 \mathrm{~nm}$ possibly making a minor contribution. ${ }^{35}$ Control pump-probe measurements with the same excitation and probe pulses as those used for 2DES also showed a positive signal after a few picoseconds (Figure S1), indicating the formation of $\mathrm{H}^{-}$. The $(810,920)$ and $(865,927) \mathrm{nm}$ cross peaks therefore reflected ET to $\mathrm{H}_{\mathrm{A}}$ being features of the $\mathrm{B}_{\mathrm{A}}^{+} \mathrm{H}_{\mathrm{A}}^{-}$and $\mathrm{P}^{+} \mathrm{H}_{\mathrm{A}}^{-} \mathrm{CT}$ states, respectively. The spectral shapes of all three cross peaks remained nearly unchanged between $10 \mathrm{ps}$ and $1 \mathrm{~ns}$. This period corresponded to decay of $\mathrm{P}^{+} \mathrm{H}_{\mathrm{A}}^{-}$to the ground state by charge recombination or via formation of the triplet state of $\mathrm{P} .{ }^{29}$

One should note that the three CT cross peaks belonged to two different ET channels. The $(815,855)$ and $(810,920) \mathrm{nm}$ ones respectively corresponded to the first and second CT state in the $\mathrm{B}^{*} \rightarrow \mathrm{P}^{+} \mathrm{B}_{\mathrm{A}}^{-} \rightarrow \mathrm{P}^{+} \mathrm{H}_{\mathrm{A}}^{-}$channel. The $(865,927) \mathrm{nm}$ one corresponded to the second $\mathrm{CT}$ state in the $\mathrm{P}^{*} \rightarrow \mathrm{P}^{+} \mathrm{B}_{\mathrm{A}}^{-} \rightarrow$ $\mathrm{P}^{+} \mathrm{H}_{\mathrm{A}}^{-}$channel. The first $\mathrm{CT}$ state expected to be at around $(855,810) \mathrm{nm}$ was not observed, mainly because its decay is faster than its formation, and furthermore, it was obscured by the P ESA. It will be revealed by global fitting, as discussed below. Comparing the amount of formed $\mathrm{H}^{-}$from the two channels, we can conclude that because $\mathrm{B}^{*} \rightarrow \mathrm{P}^{+} \mathrm{B}_{\mathrm{A}}^{-}(0.4 \mathrm{ps})$ is faster than $\mathrm{P}^{*} \rightarrow \mathrm{P}^{+} \mathrm{B}_{\mathrm{A}}^{-}(3.4 \mathrm{ps}) \mathrm{ET}$, the $\mathrm{B}^{*}$ channel is more efficient. The results show that $2 \mathrm{DES}$ cannot only track the two channels but also provides a way to quasi-quantitatively assess the relative yield of competing channels, which is almost impossible in $1 \mathrm{D}$ measurements.

To obtain the spectral features and evolution time constants of each ET species, a global analysis was applied. The fitting was started with a parallel or a sequential model, which respectively gave decay associated spectra (DAS) or evolution associated spectra (EAS). ${ }^{13,17,18,36}$ Both models gave the same characteristic time constants: 29 fs, 430 fs, 3.4 ps, and >1 ns. Both DAS or EAS may vary from the actual spectra of the true 

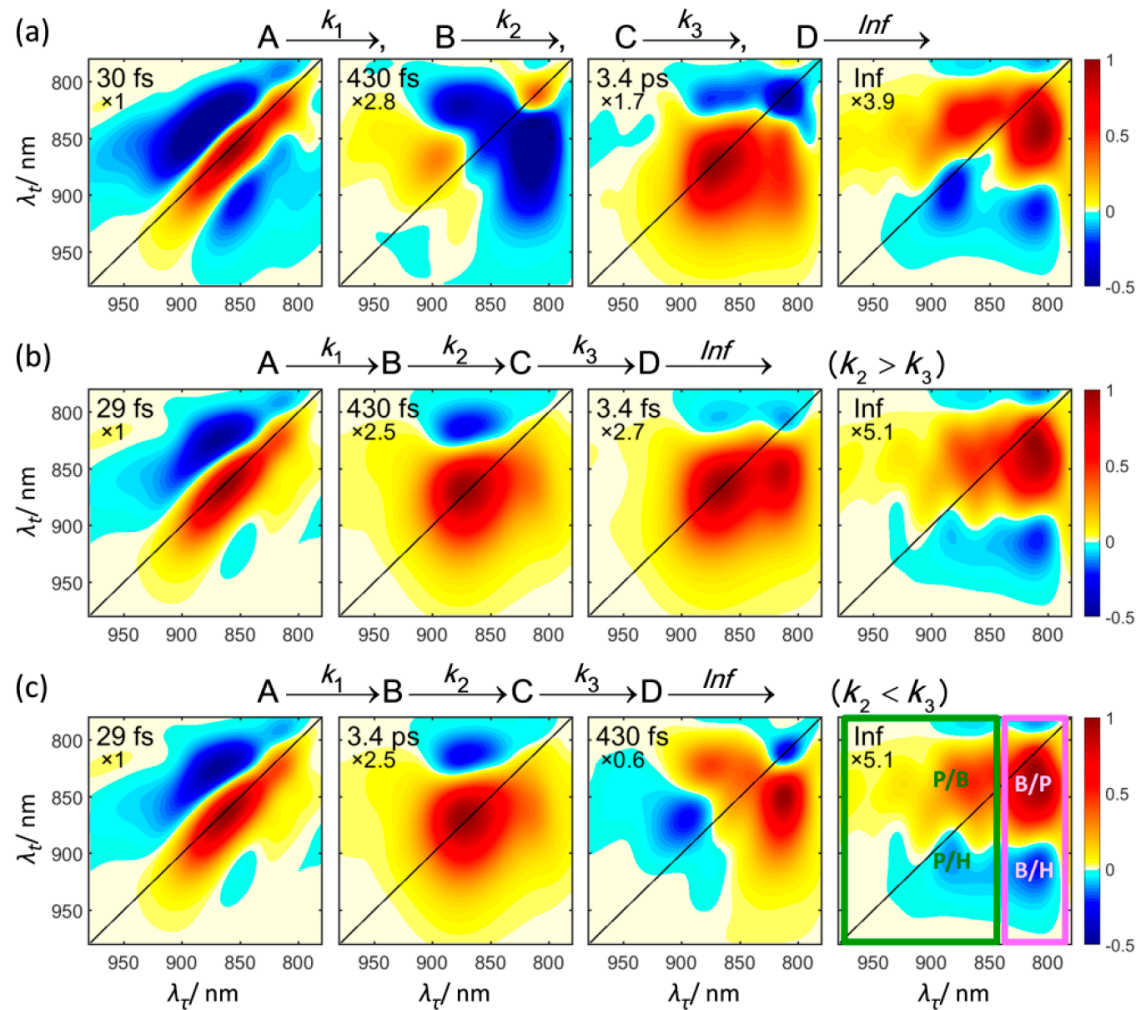

Figure 3. 2D DAS (a), EAS (b), and SAS (c) with the respective kinetic model shown on their top. The time constants of each species are shown in the left-top corner of each panel. The spectra are normalized to the maximum of the diagonal $\mathrm{P}$ signal, and the relative amplitude multiplier is shown below the time constant. The $2 \mathrm{D}$ spectral regions corresponding to the $\mathrm{P}^{*}$ and $\mathrm{B}^{*}$ channels are shown within dark green and pink windows, respectively, in the last plot of $(c)$.

species involved if the model deviates from the real dynamics through which species evolve. For example, in the present case, the DAS (Figure 3a) of species B and C did not exhibit the features that each CT species should exhibit. This discrepancy was because the real dynamic processes are principally sequential for each ET channel (Figure 3b). Furthermore, according to the well-established cascade scheme for ET in the bacterial RC, a four-component sequential model with a faster third step than second step (i.e., $k_{2}<k_{3}$ ) best agrees with the real situation of the $\mathrm{P}^{*} \mathrm{ET}$ channel (Figure $3 \mathrm{c}$ ). The spectra obtained with this model are referred to as species associated spectra (SAS). Comparison of the EAS (Figure $3 \mathrm{~b}$ ) and SAS (Figure 3c) showed that the biggest difference was for species $\mathrm{C}$, which is the second of the two species involved in the dynamic inversion. Spectra of the other species were not affected by the inversion. Furthermore, this difference was pronounced for the $\mathrm{P}^{*} \mathrm{ET}$ channel, while it had much smaller influence on the $\mathrm{B}^{*}$ channel.

Each intermediate state along the ET cascade was well resolved in the four SAS. As discussed above, the SAS model agrees with the P* ET channel; therefore, below we will focus on the $2 \mathrm{D}$ spectra region of this channel (the dark green window in Figure 3c). The first SAS corresponded to $\mathrm{P}^{*}$ at the Franck-Condon point. Its lifetime was very short, $29 \mathrm{fs}$, due to fast spectral diffusion. The second SAS contained also the GSB, SE, and ESA signals. The GSB peak was nearly symmetrically broadened along the antidiagonal direction, and its center position, $(872,869) \mathrm{nm}$, was red-shifted by about $5 \mathrm{~nm}$ along the diagonal compared to that of the first SAS, $(863,865) \mathrm{nm}$. This indicated that a new equilibrium of the excitonic state's manifold was established. An electron was then transferred from $\mathrm{P}^{*}$ to $\mathrm{B}_{\mathrm{A}}$, the 3.4 ps lifetime of $\mathrm{P}^{*}$ being determined by the $\mathrm{P}^{*} \rightarrow \mathrm{P}^{+} \mathrm{B}_{\mathrm{A}}^{-} \mathrm{CT}$ rate.

The third SAS contained positive ESA signals for $\mathrm{P}$ and $\mathrm{B}$ and two symmetrical cross peaks relative to the diagonal. The above-diagonal cross peak centered at $(870,820) \mathrm{nm}$ corresponded to $\mathrm{P}^{*} \rightarrow \mathrm{P}^{+} \mathrm{B}_{\mathrm{A}}^{-} \mathrm{ET}$, while the below-diagonal cross peak centered at $(810,855) \mathrm{nm}$ corresponded to $\mathrm{B}^{*} \rightarrow$ $\mathrm{P}^{+} \mathrm{B}_{\mathrm{A}}^{-}$ET. They both reflected the properties of the $\mathrm{P}^{+} \mathrm{B}_{\mathrm{A}}^{-} \mathrm{CT}$ state, but with different origins. We can see that the $\mathrm{P}^{+} \mathrm{B}_{\mathrm{A}}^{-} \mathrm{CT}$ state that originated from $\mathrm{P}^{*}$ was only resolved by fitting with a correct model. The third SAS had a lifetime of $430 \mathrm{fs}$, which should be determined by the $\mathrm{P}^{+} \mathrm{B}_{\mathrm{A}}^{-} \rightarrow \mathrm{P}^{+} \mathrm{H}_{\mathrm{A}}^{-}$ET rate. However, the time constant of $\mathrm{B}^{*} \rightarrow \mathrm{P}^{+} \mathrm{B}_{\mathrm{A}}^{-} \mathrm{CS}$ was also close to this value, 0.2-0.5 ps; therefore, it is difficult to distinguish the two dynamic processes.

The last SAS was more complex, containing weak GSB and ESA signals for both $\mathrm{P}$ and $\mathrm{B}$, two positive cross peaks representing $\mathrm{P}^{+} \mathrm{B}_{\mathrm{A}}^{-}$, and two negative cross peaks centered at $(810,912)$ and $(882,905) \mathrm{nm}$. The latter corresponded to the formation of $\mathrm{H}^{-}$from $\mathrm{B}^{*}$ and $\mathrm{P}^{*} \mathrm{ET}$, respectively, bearing the features of $\mathrm{B}_{\mathrm{A}}^{+} \mathrm{H}_{\mathrm{A}}^{-}$and $\mathrm{P}^{+} \mathrm{H}_{\mathrm{A}}^{-} \mathrm{CT}$ states. Thus, this SAS exhibited not only features of the $\mathrm{P}^{+} \mathrm{H}_{\mathrm{A}}^{-}$state, which is the fourth and final species, but also features of the other CT states that are preceding intermediates in the ET cascade. This SAS decayed only a little until the maximal delay time of $1 \mathrm{~ns}$ used in this work, consistent with the measured lifetime of this state in the AM260W RC of 17 ns. $^{28}$ To summarize, the sequential ET processes from $\mathrm{P}$ to $\mathrm{H}_{\mathrm{A}}$ were fully traced by $2 \mathrm{DES}$ to be $\mathrm{P}^{*} \stackrel{29 \mathrm{fs}}{\longrightarrow} \mathrm{P}_{\mathrm{A}}^{+} \mathrm{P}_{\mathrm{B}}^{-} \stackrel{3.4 \mathrm{ps}}{\longrightarrow} \mathrm{P}^{+} \mathrm{B}_{\mathrm{A}}^{-} \stackrel{430 \mathrm{fs}}{\longrightarrow} \mathrm{P}^{+} \mathrm{H}_{\mathrm{A}}^{-} \stackrel{>1 \mathrm{~ns}}{\longrightarrow}$. In accord with previous observations that $\mathrm{P}^{+} \mathrm{B}_{\mathrm{A}}^{-}$decayed much faster than it 

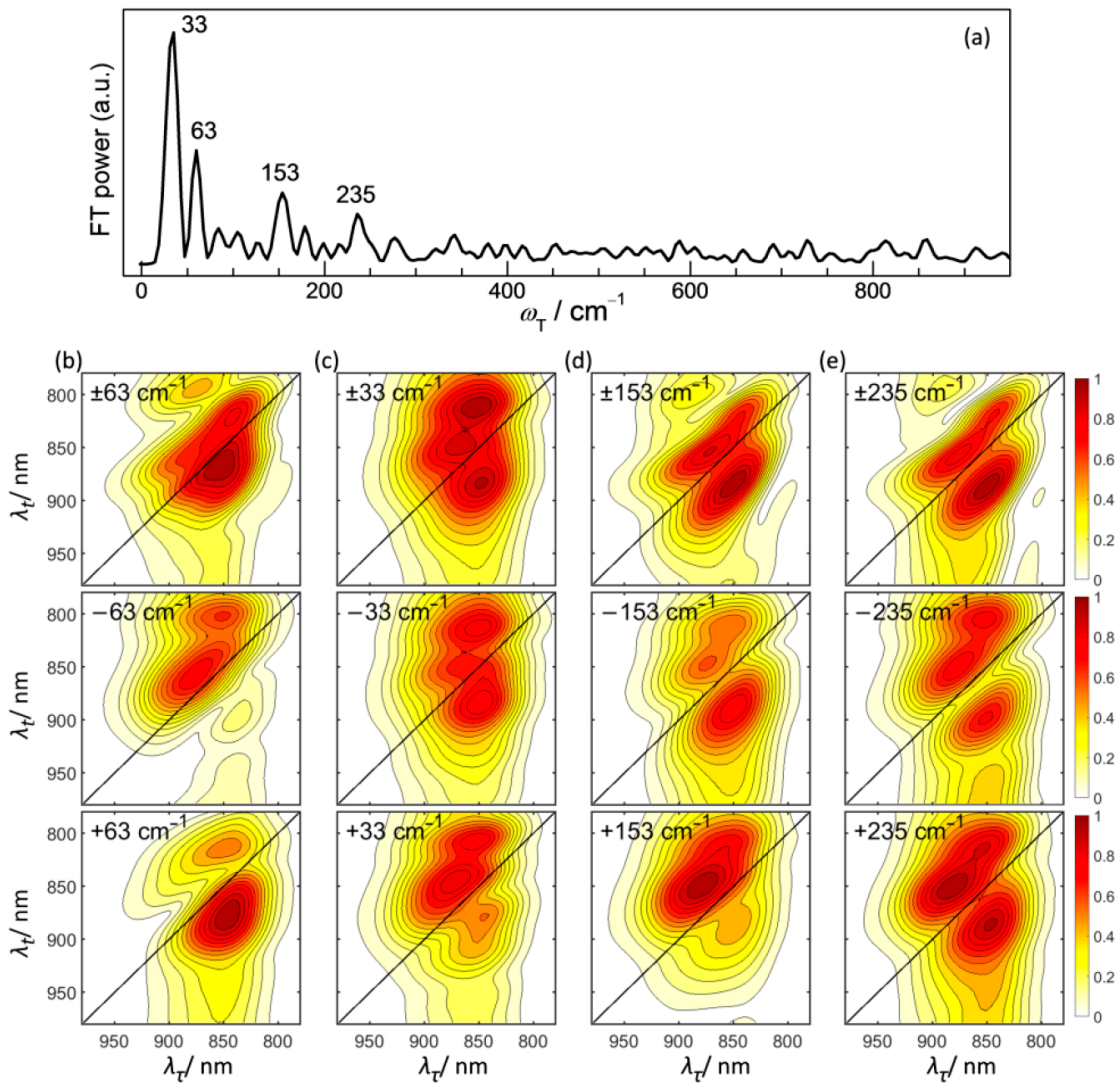

Figure 4. (a) Summary FT power spectrum of the oscillations in the real rephasing signal $(0-2 \mathrm{ps})$. (b-e) $2 \mathrm{D}$ frequency maps of the four $\omega_{\mathrm{T}}$ frequencies computed from the real-valued rephasing spectra (top) and from the complex-valued rephasing spectra $\left(\right.$ middle: $-\omega_{\mathrm{T}} ;$ bottom: $\left.+\omega_{\mathrm{T}}\right)$.

formed, it was nearly spectrally invisible in raw data and was only revealed by global fitting using the correct model.

In the $\mathrm{B}^{*}$ ET channel (the pink window in Figure 3c), the rise time constants of the $\mathrm{B} / \mathrm{P}$ and $\mathrm{B} / \mathrm{H}$ cross peaks were $430 \mathrm{fs}$ and 3.4 ps. Therefore, the dynamic evolution may be better described with EAS than SAS, although the EAS and SAS in this 2D spectral region did not exhibit essential difference. From the above results, we can see that the 2D spectra of each $\mathrm{CT}$ intermediate state exhibited more distinguishing characteristics than those that can be observed in $1 \mathrm{D}$ measurements, being an advantage of exploiting $2 \mathrm{DES}$ for complex systems.

The 2D spectra exhibited long-lived (up to $2 \mathrm{ps}$ ) oscillations. Figure 4a presents a summary Fourier transform (FT) power spectrum obtained by summing the squared absolute value (Frobenius norm) of the FTs of the $T$ traces across the 2D spectra. The prominent frequencies were 33, 63, 153, and 235 $\mathrm{cm}^{-1}$. These frequencies were consistent with those determined previously by pump-probe measurements. ${ }^{31,32}$ Also, the 33,63 , and $153 \mathrm{~cm}^{-1}$ frequencies have their correspondences in resonance Raman spectrum, ${ }^{37} 33,70$, and $145 \mathrm{~cm}^{-1}$.

To understand the origin of the oscillations, $2 \mathrm{D}$ frequency maps were analyzed. The $\pm \omega_{\mathrm{T}}$ frequency maps were yielded from the real part of the rephasing data, while the separate $-\omega_{\mathrm{T}}$ and $+\omega_{\mathrm{T}}$ frequency maps were from the full complex part. It has been shown that the characteristic patterns and symmetries in the frequency maps can help to distinguish electronic, vibrational, or mixed coherences. ${ }^{38-42}$ The $\pm 63 \mathrm{~cm}^{-1}$ frequency map (Figure $4 \mathrm{~b}$ for rephasing and Figure S2 for non-rephasing) was dominated by the diagonal peak, indicating that corresponding oscillations origined from vibrational coherence. ${ }^{39}$ The features of the -63 and $+63 \mathrm{~cm}^{-1}$ frequency maps resembled those in a carefully designed experiment for identifying vibrational coherence. ${ }^{41}$ The below-diagonal peak in the $+\omega_{\mathrm{T}}$ rephasing frequency map reflects ground-state vibrational superpositions, while the above-diagonal peak in the $-\omega_{\mathrm{T}}$ rephasing frequency map reflects excited-state vibrational superpositions. ${ }^{40,41}$ Therefore, the oscillation with $63 \mathrm{~cm}^{-1}$ could be assigned to vibrational coherence in both ground and excited states. It is notable that a peak at about $(865,805) \mathrm{nm}$ appeared in all the frequency maps and was the most pronounced in the $33 \mathrm{~cm}^{-1}$ ones. It could be from the contribution of B. Below our analysis would focus on the main P peak.

The features of the 33, 153, and $235 \mathrm{~cm}^{-1}$ frequency maps (Figure $4 c-e$ ) were different. First of all, in the $\pm \omega_{\mathrm{T}}$ rephasing frequency maps, the diagonal peak disappeared; instead, two off-diagonal peaks appeared symmetrically along the diagonal. It indicated a different coherent nature from that for the 63 $\mathrm{cm}^{-1}$ oscillation. Second, for 33 and $153 \mathrm{~cm}^{-1}$, the dominant peak was below-diagonal in the $-\omega_{\mathrm{T}}$ rephasing frequency map, while it was above-diagonal in the $+\omega_{\mathrm{T}}$ rephasing frequency map, which was opposite from the $63 \mathrm{~cm}^{-1}$ case. For $235 \mathrm{~cm}^{-1}$, the features were a bit deviated; both above- and belowdiagonal peaks with comparable amplitudes appeared in each $-\omega_{\mathrm{T}}$ and $+\omega_{\mathrm{T}}$ rephasing frequency maps. The features observed in the 33 and $153 \mathrm{~cm}^{-1}$ frequency maps can be explained by strongly coupled excited-state vibronic coherence. $^{39,42}$

Long-lived vibronic coherence was found responsible for increasing the rate of $\mathrm{EET}^{11}$ and $\mathrm{CS}^{14,15}$ in the RC. It was thought that resonant electronic-vibrational coupling can sustain coherences between electronic states, ${ }^{43,44}$ which, 
however, was doubted later. ${ }^{45}$ Very recently, the essence of vibronic coherence was proposed to be excited-state vibrational coherences shifted to the ground state as a result of a release of electronic energy during EET, which was concluded from the vibronic coherence coupled with the $\mathrm{H}^{*} \rightarrow \mathrm{B} \rightarrow \mathrm{P}$ EET process in the Rba. sphaeroides RC. ${ }^{46}$ On the basis of this mechanism, the 33 and $153 \mathrm{~cm}^{-1}$ oscillations can be assigned to excitedstate vibronic coherence followed by a shift into the groundstate vibrational coherence. The assignment of the $235 \mathrm{~cm}^{-1}$ oscillations was not as definite as the 33 and $153 \mathrm{~cm}^{-1}$ ones. It may be weakly coupled vibronic coherence.

Universal appearance of vibrational modes coupling in the CS process in RCs (both bacterial and high plant) has attracted much theoretical interest in how coupling of specific vibrational modes facilitates this process. Our previous simulation of the Rba. sphearoides RC based on Redfield theory revealed that nonequilibrated vibrational modes are involved in CS and determine the dynamics of the excited-state wavepacket. ${ }^{34}$ Two modes were identified: the $130 \mathrm{~cm}^{-1}$ one connecting with the intermolecular dynamics within $\mathrm{P}$ and the $32 \mathrm{~cm}^{-1}$ one responsible for stabilization of the primary $\mathrm{CT}$ state. Later, $\mathrm{ab}$ initio molecular dynamics simulation found that coupling with two vibrational modes, 50 and $100 \mathrm{~cm}^{-1}$, leads to unidirectional displacement of electron density to establish the CT state. ${ }^{47,48}$ Redfield theory simulation of the Photosystem II RC revealed that resonant vibrations can modify delocalization of the exciton states and thus promote direct CS. ${ }^{49,50}$

Our finding that the 153 and $33 \mathrm{~cm}^{-1}$ modes appeared as excited-state vibronic coherence matches very well with the simulation results. Furthermore, the $153 \mathrm{~cm}^{-1}$ mode, assigned to be associated with the internal CT state within $\mathrm{P},{ }^{34}$ did not appear in the 2DES measurement of the oxidized Rba. sphaeroides $\mathrm{RC}{ }^{46}$ implying that it couples to the CS process. Hence we speculate that excitation of them facilitates the CS process in the Rba. sphaeroides RC. This vibronic coherence mechanism could be responsible for the superhigh efficiency of natural photochemical CS, which may offer a blueprint for constructing photochemical devices with high energy conversion efficiencies using abundant materials.

\section{ASSOCIATED CONTENT}

\section{S Supporting Information}

The Supporting Information is available free of charge on the ACS Publications website at DOI: 10.1021/acs.jpclett.8b00108.

Experimental methods, pump-probe spectra, and nonrephasing 2D frequency maps (PDF)

\section{AUTHOR INFORMATION}

\section{Corresponding Author}

*E-mail: fma@iccas.ac.cn.

\section{ORCID}

Fei Ma: 0000-0002-7607-6429

\section{Notes}

The authors declare no competing financial interest.

\section{ACKNOWLEDGMENTS}

F.M., E.R., and R.v.G. were supported by an Advanced Investigator grant from the European Research Council (No. 267333, PHOTPROT) and the TOP-grant (700.58.305) from the Foundation of Chemical Science part of NWO. R.v.G. gratefully acknowledges his Academy Professor grant from The
Netherlands Royal Academy of Sciences (KNAW). M.R.J acknowledges support from the Biotechnology and Biological Sciences Research Council of the U.K. (Project BB/I022570/ 1).

\section{REFERENCES}

(1) van Brederode, M. E.; Jones, M. R. Reaction Centers of Purple Bacteria. Subcellular Biochemistry 35: Enzyme-catalyzed electron and radical transfer; Kluwer Academic I Plenum Publishers: New York, 2000; pp 621-676.

(2) Heathcote, P.; Jones, M. R. The Structure-Function Relationships of Photosynthetic Reaction Centers. Comprehensive Biophysics; Academic Press: Oxford, U.K., 2012; Vol. 8, pp 115-144.

(3) Feher, G.; Allen, J. P.; Okamura, M. Y.; Rees, D. C. Structure and Function of Bacterial Photosynthetic Reaction Centers. Nature 1989, $339,111-116$.

(4) Steffen, M. A.; Lao, K.; Boxer, S. G. Dielectric Asymmetry in the Photosynthetic Reaction Center. Science 1994, 264, 810-816.

(5) Zinth, W.; Wachtveitl, J. The First Picoseconds in Bacterial Photosynthesis - Ultrafast Electron Transfer for the Efficient Conversion of Light Energy. ChemPhysChem 2005, 6, 871-880.

(6) van Brederode, M. E.; van Mourik, F.; van Stokkum, I. H. M.; Jones, M. R.; van Grondelle, R. Multiple Pathways for Ultrafast Transduction of Light Energy in the Photosynthetic Reaction Center of Rhodobacter sphaeroides. Proc. Natl. Acad. Sci. U. S. A. 1999, 96, 2054-2059.

(7) van Brederode, M. E.; van Grondelle, R. New and Unexpected Routes for Ultrafast Electron Transfer in Photosynthetic Reaction Centers. FEBS Lett. 1999, 455, 1-7.

(8) Brixner, T.; Mančal, T.; Stiopkin, I. V.; Fleming, G. R. PhaseStabilized Two-Dimensional Electronic Spectroscopy. J. Chem. Phys. 2004, 121, 4221-4236.

(9) Brixner, T.; Stenger, J.; Vaswani, H. M.; Cho, M.; Blankenship, R. E.; Fleming, G. R. Two-Dimensional Spectroscopy of Electronic Couplings in Photosynthesis. Nature 2005, 434, 625-628.

(10) Collini, E.; Wong, C. Y.; Wilk, K. E.; Curmi, P. M. G.; Brumer, P.; Scholes, G. D. Coherently Wired Light-Harvesting in Photosynthetic Marine Algae at Ambient Temperature. Nature 2010, 463, 644-648.

(11) Westenhoff, S.; Paleček, D.; Edlund, P.; Smith, P.; Zigmantas, D. Coherent Picosecond Exciton Dynamics in a Photosynthetic Reaction Center. J. Am. Chem. Soc. 2012, 134, 16484-16487.

(12) Schlau-Cohen, G. S.; Ishizaki, A.; Calhoun, T. R.; Ginsberg, N. S.; Ballottari, M.; Bassi, R.; Fleming, G. R. (2012) Elucidation of the Timescales and Origins of Quantum Electronic Coherence in LHCII. Nat. Chem. 2012, 4, 389-395.

(13) Ostroumov, E. E.; Mulvaney, R. M.; Cogdell, R. J.; Scholes, G. D. Broadband 2D Electronic Spectroscopy Reveals a Carotenoid Dark State in Purple Bacteria. Science 2013, 340, 52-56.

(14) Romero, E.; Augulis, R.; Novoderezhkin, V. I.; Ferretti, M.; Thieme, J.; Zigmantas, D.; van Grondelle, R. Quantum Coherence in Photosynthesis for Efficient Solar-Energy Conversion. Nat. Phys. 2014, 10, 676-682.

(15) Fuller, F. D.; Pan, J.; Gelzinis, A.; Butkus, V.; Senlik, S. S.; Wilcox, D. E.; Yocum, C. F.; Valkunas, L.; Abramavicius, D.; Ogilvie, J. P. Vibronic Coherence in Oxygenic Photosynthesis. Nat. Chem. 2014, 6, 706-711.

(16) Chenu, A.; Scholes, G. D. Coherence in Energy Transfer and Photosynthesis. Annu. Rev. Phys. Chem. 2015, 66, 69-96.

(17) Dostál, J.; Pšenčík, J.; Zigmantas, D. In situ Mapping of the Energy Flow through the Entire Photosynthetic Apparatus. Nat. Chem. 2016, 8, 705-710.

(18) Ma, F.; Yu, L.; Hendrikx, R.; Wang-Otomo, Z.; van Grondelle, R. Direct Observation of Energy Detrapping in LH1-RC Complex by Two-Dimensional Electronic Spectroscopy. J. Am. Chem. Soc. 2017, 139, 591-594.

(19) Ma, F.; Yu, L.; Hendrikx, R.; Wang-Otomo, Z.; van Grondelle, R. Excitonic and Vibrational Coherence in the Excitation Relaxation 
Process of Two LH1 Complexes as Revealed by Two-Dimensional Electronic Spectroscopy. J. Phys. Chem. Lett. 2017, 8, 2751-2756.

(20) Romero, E.; Novoderezhkin, V. I.; van Grondelle, R. Quantum Design of Photosynthesis for Bio-Inspired Solar-Energy Conversion. Nature 2017, 543, 355-365.

(21) Fingerhut, B. P.; Mukamel, S. Resolving the Electron Transfer Kinetics in the Bacterial Reaction Center by Pulse Polarized 2-D Photon Echo Spectroscopy. J. Phys. Chem. Lett. 2012, 3, 1798-1805.

(22) Lee, H.; Cheng, Y.; Fleming, G. R. Coherence Dynamics in Photosynthesis: Protein Protection of Excitonic Coherence. Science 2007, 316, 1462-1465.

(23) Schlau-Cohen, G. S.; De Re, E.; Cogdell, R. J.; Fleming, G. R. Determination of Excited-State Energies and Dynamics in the B Band of the Bacterial Reaction Center with 2D Electronic Spectroscopy. J. Phys. Chem. Lett. 2012, 3, 2487-2492.

(24) Flanagan, M. L.; Long, P. D.; Dahlberg, P. D.; Rolczynski, B. S.; Massey, S. C.; Engel, G. S. Mutations to R. sphaeroides Reaction Center Perturb Energy Levels and Vibronic Coupling but Not Observed Energy Transfer Rates. J. Phys. Chem. A 2016, 120, 1479-1487.

(25) Ma, F.; Swainsbury, D. J. K.; Jones, M. R.; van Grondelle, R. Photoprotection through Ultrafast Charge Recombination in Photochemical Reaction Centers under Oxidizing Conditions. Philos. Trans. R. Soc., B 2017, 372, 20160378.

(26) Ridge, J. P.; van Brederode, M. E.; Goodwin, M. G.; van Grondelle, R.; Jones, M. R. Mutations that Modify or Exclude Binding of the $\mathrm{Q}_{\mathrm{A}}$ Ubiquinone and Carotenoid in the Reaction Center from Rhodobacter sphaeroides. Photosynth. Res. 1999, 59, 9-26.

(27) McAuley, K. E.; Fyfe, P. K.; Ridge, J. P.; Cogdell, R. J.; Isaacs, N. W.; Jones, M. R. Ubiquinone Binding, Ubiquinone Exclusion, and Detailed Cofactor Conformation in a Mutant Bacterial Reaction Center. Biochemistry 2000, 39, 15032-15043.

(28) Gibasiewicz, K.; Pajzderska, M.; Potter, J. A.; Fyfe, P. K.; Dobek, A.; Brettel, K.; Jones, M. R. Mechanism of Recombination of the $\mathrm{P}^{+} \mathrm{H}_{\mathrm{A}}{ }^{-}$Radical Pair in Mutant Rhodobacter sphaeroides Reaction Centers with Modified Free Energy Gaps between $\mathrm{P}^{+} \mathrm{B}_{\mathrm{A}}^{-}$and $\mathrm{P}^{+} \mathrm{H}_{\mathrm{A}}{ }^{-}$. J. Phys. Chem. B 2011, 115, 13037-13050.

(29) Pawlowicz, N. P.; van Grondelle, R.; van Stokkum, I. H. M.; Breton, J.; Jones, M. R.; Groot, M. L. Identification of the First Steps in Charge Separation in Bacterial Photosynthetic Reaction Centers of Rhodobacter sphaeroides by Ultrafast Mid-Infrared Spectroscopy: Electron Transfer and Protein Dynamics. Biophys. J. 2008, 95, $1268-1284$.

(30) Vos, M. H.; Rappaport, F.; Lambry, J. C.; Breton, J.; Martin, J. L. Visualization of Coherent Nuclear Motion in a Membrane Protein by Femtosecond Spectroscopy. Nature 1993, 363, 320-325.

(31) Vos, M. H.; Jones, M. R.; Hunter, C. N.; Breton, J.; Lambry, J.; Martin, J. Coherent Dynamics during the Primary Electron-Transfer Reaction in Membrane-Bound Reaction Centers of Rhodobacter sphaeroides. Biochemistry 1994, 33, 6750-6757.

(32) Vos, M. H.; Jones, M. R.; Breton, J.; Lambry, J. C.; Martin, J. L. Vibrational Dephasing of Long- and Short-lived Primary Donor Excited States in Mutant Reaction Centers of Rhodobacter sphaeroides. Biochemistry 1996, 35, 2687-2692.

(33) Yakovlev, A. G.; Jones, M. J.; Potter, J. A.; Fyfe, P. K.; Vasilieva, L. G.; Shkuropatov, A. Y.; Shuvalov, V. A. Primary Charge Separation between $\mathrm{P}^{*}$ and $\mathrm{B}_{\mathrm{A}}$ : Electron-Transfer Pathways in Native and Mutant GM203L Bacterial Reaction Centers. Chem. Phys. 2005, 319, 297307.

(34) Novoderezhkin, V. I.; Yakovlev, A. G.; van Grondelle, R; Shuvalov, V. A. Coherent Nuclear and Electronic Dynamics in Primary Charge Separation in Photosynthetic Reaction Centers: A Redfield Theory Approach. J. Phys. Chem. B 2004, 108, 7445-7457.

(35) Zhu, J.; van Stokkum, I. H. M.; Paparelli, L.; Jones, M. R.; Groot, M. L. Early Bacteriopheophytin Reduction in Charge Separation in Reaction Centers of Rhodobacter sphaeroides. Biophys. J. 2013, 104, 2493-2502.

(36) van Stokkum, I. H. M.; Larsen, D. S.; van Grondelle, R. Global and Target Analysis of Time-Resolved Spectra. Biochim. Biophys. Acta, Bioenerg. 2004, 1657, 82-104.
(37) Cherepy, N. J.; Shreve, A. P.; Moore, L. J.; Boxer, S. G.; Mathies, R. A. Temperature Dependence of the $\mathrm{Q}_{\mathrm{y}}$ Resonance Raman Spectra of Bacteriochlorophylls, the Primary Electron Donor, and Bacteriopheophytins in the Bacterial Photosynthetic Reaction Center. Biochemistry 1997, 36, 8559-8566.

(38) Butkus, V.; Zigmantas, D.; Valkunas, L.; Abramavicius, D. Vibrational vs. Electronic Coherences in 2D Spectrum of Molecular Systems. Chem. Phys. Lett. 2012, 545, 40-43.

(39) Butkus, V.; Zigmantas, D.; Abramavicius, D.; Valkunas, L. Distinctive Character of Electronic and Vibrational Coherences in Disordered Molecular Aggregates. Chem. Phys. Lett. 2013, 587, 93-98.

(40) Seibt, J.; Pullerits, T. Beating Signals in 2D Spectroscopy: Electronic or Nuclear Coherences? Application to a Quantum Dot Model System. J. Phys. Chem. C 2013, 117, 18728-18737.

(41) de A. Camargo, F. V.; Grimmelsmann, L.; Anderson, H. L.; Meech, S. R.; Heisler, I. A. Resolving Vibrational from Electronic Coherences in Two-Dimensional Electronic Spectroscopy: The Role of the Laser Spectrum. Phys. Rev. Lett. 2017, 118, 033001.

(42) Butkus, V.; Alster, J.; Bašinskaite, E.; Augulis, R.; Neuhaus, P.; Valkunas, L.; Anderson, H. L.; Abramavicius, D.; Zigmantas, D. Discrimination of Diverse Coherences Allows Identification of Electronic Transitions of a Molecular Nanoring. J. Phys. Chem. Lett. 2017, 8, 2344-2349.

(43) Chin, A. W.; Prior, J.; Rosenbach, R.; Caycedo-Soler, F.; Huelga, S. F.; Plenio, M. B. The Role of Non-Equilibrium Vibrational Structures in Electronic Coherence and Recoherence in PigmentProtein Complexes. Nat. Phys. 2013, 9, 113-118.

(44) Tiwari, V.; Peters, W. K.; Jonas, D. M. Electronic Resonance with Anticorrelated Pigment Vibrations Drives Photosynthetic Energy Transfer outside the Adiabatic Framework. Proc. Natl. Acad. Sci. U. S. A. 2013, 110, 1203-1208.

(45) Duan, H. G.; Prokhorenko, V. I.; Cogdell, R. J.; Ashraf, K.; Stevens, A. L.; Thorwart, M.; Miller, R. J. D. Nature Does Not Rely on Long-Lived Electronic Quantum Coherence for Photosynthetic Energy Transfer. Proc. Natl. Acad. Sci. U. S. A. 2017, 114, 8493-8498.

(46) Paleček, D.; Edlund, P.; Westenhoff, S.; Zigmantas, D. Quantum Coherence as a Witness of Vibronically Hot Energy Transfer in Bacterial Reaction Center. Sci. Adv. 2017, 3, e1603141.

(47) Eisenmayer, T. J.; de Groot, H. J. M.; van de Wetering, E.; Neugebauer, J.; Buda, F. (2012) Mechanism and Reaction Coordinate of Directional Charge Separation in Bacterial Reaction Centers. J. Phys. Chem. Lett. 2012, 3, 694-697.

(48) Eisenmayer, T. J.; Lasave, J. A.; Monti, A.; de Groot, H. J. M.; Buda, F. Proton Displacements Coupled to Primary Electron Transfer in the Rhodobacter sphaeroides Reaction Center. J. Phys. Chem. B 2013, $117,11162-11168$.

(49) Novoderezhkin, V. I.; Romero, E.; van Grondelle, R. How Exciton-Vibrational Coherences Control Charge Separation in the Photosystem II Reaction Center. Phys. Chem. Chem. Phys. 2015, 17, 30828-30841.

(50) Novoderezhkin, V. I.; Romero, E.; Prior, J.; van Grondelle, R. Exciton-Vibrational Resonance and Dynamics of Charge Separation in the Photosystem II Reaction Center. Phys. Chem. Chem. Phys. 2017, 19, 5195-5208. 